\section{Amyotrophic lateral sclerosis: one or multiple causes?}

\author{
Aline Furtado Bastos, 1 Marco Orsini,2,3 \\ Dionis Machado, 4 \\ Mariana Pimentel Mello, ${ }^{1}$ Sergio Nader, 5 \\ Júlio Guilherme Silva,2,6 \\ Antonio M. da Silva Catharino, 7 Marcos \\ R.G. de Freitas, ${ }^{2}$ Alessandra Pereira, ${ }^{8}$ \\ Luciane Lacerda Pessoa, 8 \\ Flavio R. Sztajnbok, 8 \\ Marco Araújo Leite, 2 \\ Osvaldo J.M. Nascimento, 2 \\ Victor Hugo Bastos9 \\ 1Serra dos Órgãos University, \\ Teresópolis, Rio de Janeiro; ${ }^{2}$ Federal \\ Fluminense University, Rio de Janeiro; \\ 3Medicine Department, Grande Rio \\ University, Rio de Janeiro; \\ 4Federal University of the Jequitinhonha \\ and Mucuri Valleys, Diamantina, Minas \\ Gerais;
}

${ }^{5}$ Rio de Janeiro Federal University, Rio de Janeiro;

6Masters Program in Rehabilitation

Centro Universitario Augusto Motta;

7Nova Iguaçu University, Rio de Janeiro;

${ }^{8}$ Grande Rio University (Medicine

Department);

9Federal University of the Jequitinhonha and Mucuri, Diamantina, Minas Gerais, Brazil

\section{Abstract}

The Amyotrophic lateral sclerosis (ALS) is the most common form of motor neuron disease in the adulthood, and it is characterized by rapid and progressive compromise of the upper and lower motor neurons. The majority of the cases of ALS are classified as sporadic and, until now, a specific cause for these cases still is unknown. To present the different hypotheses on the etiology of ALS. It was carried out a search in the databases: Bireme, Scielo and Pubmed, in the period of 1987 to 2011, using the following keywords: Amyotrophic lateral sclerosis, motor neuron disease, etiology, causes and epidemiology and its similar in Portuguese and Spanish. It did not have consensus as regards the etiology of ALS. Researches demonstrates evidences as regards intoxication by heavy metals, environmental and occupational causes, genetic mutations (superoxide dismutase 1), certain viral infections and the accomplishment of vigorous physical activity for the development of the disease. There is still no consensus regarding the involved factors in the etiology of ALS. In this way, new research about these etiologies are necessary, for a better approach of the patients, promoting preventive programs for the disease and improving the quality of life of the patients.

\section{Introduction}

Amyotrophic lateral sclerosis (ALS) is the most common form of motor neuron disease in adulthood and it is characterized by progressive and degenerative compromise of the upper and lower motor neurons, 1,2 resulting in progressive amyotrophy, fasciculations, paresis, and spasticity. ${ }^{3}$ Respiratory muscles failure is generally the fatal event, occurring in 1-5 years after the first manifestations of the disease. ${ }^{4}$ Its incidence is $1-3$ cases $/ 100,000$ inhabitants, and its prevalence is $3-5 / 100,000$. However, some areas in the Western Pacific (Guam Island, Kii Peninsula in Japan and Western New Guinea) present prevalence around 50 times higher. ${ }^{2,5}$ Most ALS cases are classified as sporadic, while in around 5\% there is evidence of family history (familial ALS). Commonly these cases show Mendelian autosomal dominant inheritance. However, autosomal recessive patterns have also been identified. ${ }^{1}$ Clinically and pathologically, sporadic and familial forms are similar, which suggests a common pathogenesis. ${ }^{6}$ No specific cause for most cases is known yet and unfortunately, up to this moment, there is no medication to interrupt the course of the disease. 7,8 Taking into consideration the difference in prevalence in distinct parts of the world, the absence of a defined inheritance pattern and a high prevalence of ALS in particular familial nuclei, the cause of this disease is not accurately known. The objective of this study is to present, based on literature, the different hypotheses on ALS's etiology.

\section{Materials and Methods}

This review article used articles in the data bases Bireme, Scielo and Pubmed, using the following keywords: Amyotrophic Lateral Sclerosis, motor neuron disease, etiology, causes and epidemiology, and their correspondents in Portuguese and Spanish, in the period of 1987-2011.

\section{Literature review}

\section{Genetic mutations}

In 1993, Rosen et al. ${ }^{9}$ described mutations in the codification of the gene superoxide dismutase 1 (SOD1), which catalyses the dismutation of the superoxide radical into hydrogen
Correspondence: Victor Hugo Bastos, Physical Therapy Departament, Vales do Jequitinhonha e Mucuri Federal University. Rodovia MGT 367, 5000, Alto da Jacuba, CEP 39100-000, Diamantina, Brazil.

Tel. +55.38.35321239.

E-mail: victorhugobastos@yahoo.com.br

Key words: motor neuron disease, neuromuscular Diseases, amyotrophic lateral sclerosis, etiology, hypothesis, review.

Received for publication: 1 April 2011. Accepted for publication: 2 April 2011.

This work is licensed under a Creative Commons Attribution 3.0 License (by-nc 3.0).

(C) Copyright A. Furtado Bastos et al., 2011

Licensee PAGEPress, Italy

Neurology International 2011; 3:e4

doi:10.4081/ni.2011.e4

and oxygen peroxide. SOD1 is a cytoplasmatic enzyme dependent on copper and zinc. There are more than 100 different mutations of SOD1, the most common being the substitution of valine with alanine in position 4 (A4V), most of which are dominant, except for the substitution of alanine with aspartate in position 90 (D90A), which can be recessive or dominant. Dominant mutations are believed to act with a toxic gain of function as the result of an abnormal accumulation of products of the protein S0D1.2,10,11 S0D1's toxicity suggests an oxidative damage, and an SOD1 mutation may increase motoneurons' vulnerability, inhibiting the activity of certain proteins, mainly in cases of familial ALS. Intracellular aggregation may limit mitochondrial function and disorganize neurofilamental structure, which can activate apoptotic factors and inhibit axonal transportation, respectively. In addition, similar damages to astrocytes may interrupt glutamatergic neurotransmission, resulting in excitotoxicity, with resulting cellular death. 3 Sporadic ALS differs from familial ALS in some aspects. In sporadic ALS symptoms' onset usually occur around the age of 55-65 years, with a mean of 64 years; it is more prevalent in men than in women (1.5:1), probably due a hormonal protection in women and a greater exposure among men to supposed risk factors; it also presents a mortality of 1.84 per 100,000 inhabitants. ${ }^{12}$ However, Orsini et al. ${ }^{13}$ report 3 cases of sporadic ALS of juvenile onset. In this series of cases, the age of the individuals varied between 26-28 years, none presented family history of the disease. Differently, in familial ALS the age of onset of symptoms varies between $45-55$ years, and the prevalence is similar between men and women, with a lower life expectancy. Both forms of the disease are similar in clinical and 
pathological presentation.2 Various genetic mutations found can be considered to act in the predisposition to ALS, including one which regulates retrograde axonal transportation. ${ }^{8}$ Other responsible genes for familial ALS include alsin (ALS2), ${ }^{14,15}$ senataxin (ALS4), ${ }^{16}$ angiogenin, 17 and a mutation in the subunit p150 of dynactin (DCTN1).18,19 Despite numerous researches on SOD1, this mutation can be identified in around $20 \%$ of cases. 9,20 The other $80 \%$ of familial cases are related with genes not yet known. Sporadic ALS may possibly be related to alterations in more complex genetic systems, in which those would act more as risk factors than as a direct cause for the disease. ${ }^{8}$ Due to the clinic similarity with the sporadic form, progresses in the elucidation of the mechanisms involved in the familial form may favor researches in both forms of the disease. ${ }^{3}$ Currently, mutations in the FUS gene have been described as the cause of familial ALS. ${ }^{21}$ Rademakers et al.22 developed a genetic analysis of FUS in 200 ALS patients, 32 familial type cases, and 168 sporadic. A mutation was identified in a familial case (p.R521C) and another one in a sporadic case (p.G187S). This study suggests that the phenotype with FUS mutations goes beyond ALS classical form, indicating that there are clinical genetic correlations specific to the case. TARDBP- related ALS appears indistinguishable from ALS of other known and unknown causes based on gender ratio, age of onset, symptom distribution, and severity of disease, and is characterized by lower motor neuron (LMN) and upper motor neuron (UMN) involvement. More than 80 persons with TARDBP- related ALS have been described in the literature. The spectrum of the clinical aspects in these patients appears to overlap significantly with idiopathic and SOD1-related ALS. It's inherited in an autosomal dominant manner. The proportion of cases caused by new mutations is unknown. Each child of an individual with TARDBP-related ALS has a $50 \%$ chance of inheriting the mutation. ${ }^{23}$ The diagnosis is established when a pathogenic TARDBP mutation is identified in a patient meeting clinical diagnostic criteria for ALS. Spinal onset was reported in $77 \%$ of individuals with TARDBP mutations and (LMN) predominant disease was seen in $39 \%$ [Kühnlein et al. 2008]. Frontotemporal dementia (FTD)and cognitive impairments have not been reported in individuals with TARDBP mutations.24 After discovery the mutations in the gene coding for the $\mathrm{Cu} / \mathrm{Zn}$ superoxide dismutase 1 (SOD1) in subsets of familial ALS patients, several transgenic mouse studies have been generated with various forms of SOD1 mutants overexpressed at different levels. Studies with these mice yielded complex results with multiple targets of damage in disease including mitochondria, proteasomes, and secretory pathways.
Evidence is emerging of a complex interaction between genetic and molecular factors, with resultant damage of critical target proteins and organelles within the motor neuron. Results revealed that neurofilament heavy subunit was identified in $70 \%$ of ALS cases and conclude that this subunit may be a promising biomarker for clinical diagnosis of ALS. ${ }^{25,26}$

Environmental and Occupational Causes

The possibility of environmental factors being involved in ALS etiology is due to variations in its incidence and to the fact that some genetic and epidemiologic studies associate it with the exposure to pesticides. ${ }^{27-29}$ In this context, the high prevalence of ALS cases in the Guam region can be cited, when compared with the rest of the world. In this region, a complex clinical picture constituted by ALS, Parkinsonism and dementia (ALS-PD complex) occurred in the Chamorro population, with prevalence around 50 times higher than in the West.2,5 Three great theories were proposed to justify the high incidence in this population: genetic, because of the familial aggregation of ALS cases and ALS-PD complex; food consumption of a neurotoxin from the plant cycad; and exposure to potable water and cultivation soils with low calcium and high aluminum. ${ }^{11}$ However, according to a new theory, the high rates of amyotrophic lateral sclerosis (ALS) on Guam may have been caused by the native people's predilection for eating bats. Researchers proposed the theory based partly on observations that the bats, a delicacy among native Guamanians, eat poisonous nuts from the cycad tree, a sort of palm tree indigenous to the island. ${ }^{30}$ Actually, Beta-methyl-amino-Lalanine, (BMAA), is found in multiple components of the traditional Chamorro diet of Guam and this confounds epidemiological analysis based on a single dietary item. Recent discoveries that found that BMAA is produced by symbiotic cyanobacteria within specialized roots of the cycads; that the concentration of protein-bound BMAA is up to a hundred-fold greater than free BMAA in the seeds and flour; that various animals forage on the seeds (flying foxes, pigs, deer), leading to biomagnification up the food chain in Guam; and that protein-bound BMAA occurs in the brains of Guamanians dying of ALS/PDC (average concentration $627 \mathrm{microg} / \mathrm{g}, 5 \mathrm{mM}$ ) but not in control brains have rekindled interest in BMAA as a possible trigger for Guamanian ALS/PDC. 31,32 Recent studies have demonstrated a decrease in the incidence of ALS and ALS-PD cases throughout the last 40 years, despite the discretion of this reduction in the decades of 1980-1990 with respect to the ALS-PD complex. 33-35 In this population, it was observed that the traditional diet included the ingestion of a type of bat, the flying fox. However, this species, due to its feeding habits, presents high concentrations of $\beta$ - $N$-methylamino-L-alanine
(BMMA), a strong excitotoxic amino acid capable of damaging the motor cortex of monkeys' brain. ${ }^{11,36}$ Some occupations or exposures seem related to the risk of ALS, including agriculture and the production of chemical products, exposure to electromagnetic fields, welding, labor or electric shocks. Aiming at assessing the relation between occupational causes and ALS mortality, Weisskopf et al. ${ }^{37}$ conducted a study in the period of 1989-2002, in which a questionnaire concerning the occupation of participants was used; they quantified the total of deaths during the period of the research. As a result, they obtained an increase of mortality among men who worked as laboratory programmers and technicians; and, among women, an increase in mortality among machine setters and nurses. The authors highlight that these results were based on a small number of cases in each professional category and that they must be interpreted with caution. In addition, for laboratory programmers and technicians, the results were not consistent between men and women, and this inconsistency might be due to men and women performing different functions within a particular work group, an aspect that was not investigated. A study conducted by Morahan et al. ${ }^{38}$ investigated the action of environmental toxins in the development of ALS in Australian population. A total of 179 sporadic ALS patients were evaluated through a questionnaire addressing issues such as exposure to metals, solvents or chemical products and residence in rural areas. The evaluation pointed towards an association of the disease with the exposure to chemical products/solvents, as well as to herbicides/pesticides, indicating that environmental toxins may be risk factors. Weisskopf et al. ${ }^{29}$ conducted a prospective and cross-sectional study, in which they observed the association between exposure to chemical agents and ALS development, through the application of questionnaires evaluating the exposure to chemical agents throughout life, and identified the number of deaths during the researched period (1989-2004). The study indicated small evidence of association between exposure to pesticides and herbicides and the emergence of ALS. However, there was a greater evidence of ALS risk with exposure to formaldehyde. Another risk factor for ALS is smoking. Cigarettes contain toxic agents which induce oxidative stress in the organism. As demonstrated by Weisskopf et al., ${ }^{39}$ who investigated the association between smoking and ALS through questionnaires, there seems to be an association of recent smoking with the increase in death due to ALS in women, but it may be associated to other factors, including hormonal factors or the use of contraceptives. A study conducted by Fang et al. ${ }^{40}$ analyzed the association between smoking, snuff dipping, and the risk of ALS incidence in a group of 
Swedish construction workers. Questionnaires on smoking habits were applied and an interview with each participant was performed to obtain information on smoking and snuff dipping. From the results no increase of ALS among smoking men or snuff dippers was observed when compared to nonsmokers. Sutedja et al. ${ }^{41}$ applied a questionnaire in 364 patients in order to investigate the independent effect of smoking, education and occupation in the development of ALS. The authors concluded that all assessed aspects are risk factors for ALS. Smokers, those with low educational level and women performing craftwork present a higher risk of developing the disease. Smokers are also more likely to develop ALS, and this was the only factor to present an independent relation with ALS. Popat et $a l .42$ investigated another risk factor for ALS: anti-inflammatory medications. They performed a controlled study, in which participated 3 million members of the Kaiser Permenente Medical Care Program, in Northern California in the period of 1996-2000. It was observed that the use of non-steroid anti-inflammatory medications had no association with ALS development, neither as a risk factor, nor as a protective factor. However, in the placebo group a difference between sexes was observed, with a higher risk to men when compared to women. Among the various sporadic forms, a number of risk factors and associations have been linked to ALS development, mainly those involving traumas (mechanic, electric, and/or surgical). A recent study describes an ALS case subsequent to an electric trauma. The 25-year-old patient suffered an intense electric shock when his left forearm had contact with an uncovered wire. There was no loss of consciousness, although small burnings were evident. Within 1 year, the patient reported muscular pains with involuntary contractions in higher limbs. Afterwards, he developed muscular weakness and fatigue, predominantly in the left hand in the beginning, with further evolution to the right hand. No bulbar muscular weakness was observed, neither of the musculature. Several complementary exams were performed, resulting in ALS diagnosis. However, the authors suggested that this case may have occurred due to an interaction between genetic factors (SOD1 mutations) and environmental factors (electric shock). ${ }^{43}$

\section{Heavy metals intoxication}

Heavy metals exposure is referred as a risk factor for the development of ALS. ${ }^{37}$ Kamel $e t$ $a l .45$ conducted a study to investigate the association of lead exposure with ALS appearance, using questionnaires and biological markers. A higher risk related to the report of occupational exposure to lead was observed, and not to a home and/or recreative exposure. An asso- ciation with high levels of blood or bone lead was also observed. Oh et al. ${ }^{46}$ described the case of an ALS patient who worked in an electronic components factory with evidence of excessive acute exposure to lead. The study concluded that, although no other assessments were performed (genetic, environmental or biologic), the probable etiology for ALS in this case was exposure to lead. Similarly, acute and chronic intoxication with mercury may cause ALS syndrome. Some authors propose that many ALS patients report a previous exposure to mercury. ${ }^{11}$ Schwarz et al. ${ }^{47}$ report a case in which a nurse developed ALS after an accident with mercury. The mercury thermometer was broken while being held by the nurse, with resulting mercury infiltration in her skin, hindering the complete surgical removal of all the particles. Three and a half years later, the 38year-old patient started to present a pronounced weakness in the lower limbs, cerebellar ataxia, fasciculation, hyperreflexia and Babinski sign. She was diagnosed with ALS, possibly explained by the slow accumulation of mercury in the SNC, since the multiple traumas resulting from the surgery may have favored retrograde axonal transportation. Recent studies suggest that copper deficiency syndrome should be included in the motor neuron diseases. Weihl et al. ${ }^{48}$ reported three cases of patients who presented copper deficiency and clinical findings of ALS including muscular atrophy and weakness and electromyographic signals of denervation and had no ALS diagnosis, due to the presence of a hypoesthesia. Excessive exposure to selenium (Se) has also been reported among the possible etiologies for ALS, with its unknown origin and indicative of severe prognosis. This relation has been based on epidemiological investigations which demonstrate an increase of ALS risk associated with the residency in a Seimpregnated area or with the consumption of water with high inorganic levels of this metal. Researches in animals have shown that Se, mainly in its inorganic form, presents a selective toxicity to motor neurons, with the compromise of muscular function. 49

\section{Viral Infections}

Persistent viral infections were also pointed as a predisposing factor for the development of ALS. ${ }^{1}$ The persistent infection by enterovirus has been reported as causing sporadic ALS, given the poliovirus's tropism by motor neurons. These infections are lyseless and non cytopathic. ${ }^{50,51}$ Some viral properties, susceptibility factors of the host and the time of exposure may be important factors for the establishment of the infection. Tropism for motor neurons, controlled by the conformation of the viral capsid and surface receptors of the host cell, guarantees that the infection select the motor neurons and remain isolated. The infec- tion may have access through the hemato-cellular barrier. Once inside, it contiguously spreads. Horizontal propagation includes crossing the middle line of the encephalic trunk or the spinal marrow, due to the seeming proximity of the anterior horns and encephalic trunk nuclei. Vertical propagation or transsynaptic propagation provokes a leap between lower and higher motor neurons, the latter leading to long distance propagations. Because the infection is persistent and propagation through motor system is successive, progression is linear instead of accelerated. Due to the variation of biologic factors, such as vital charge, virulence, and host cell factors, progression rates are highly variable among patients. Since the infection propagates, apoptosis starts, a well established capacity in motor neurons known for its complex interaction with viruses. Given the motor neurons' limitation in number and their incapacity of division, cellular death is accumulated, clinically manifesting progressive weakness, which starts in a centralized manner, spreads itself and linearly progresses. ${ }^{50}$ Studies demonstrated a possible association between persistent infection due to enterovirus and ALS development in 46 individuals. They concluded that the rate of $88.3 \%$ of nucleic acid detection of the enterovirus in the neuron's body of the spinal marrow in ALS patients suggests a strong association between both conditions. ${ }^{52}$ Some retroviruses were also described in association with ALS-like syndromes, since motor neurons' syndromes may be associated with HIV and HTLV-1, retroviruses subtypes. ${ }^{51}$ HIV is not a neurotropic virus. It rarely infects neurons, but emerges predominantly in microglias of the central nervous system. Thus, selective damages may occur to motor neurons by neurotoxic viral proteins or cytokines (e.g., COX215) and chemokines produced as a consequence of the viral infection.53 Zoccolella $e t$ $a l .54$ reported a case of a 44 -year-old man who developed ALS concomitantly to the diagnosis of HIV infection. The patient presented a drastic reduction in plasmatic levels of HIV-RNA, which could suggest the association of both conditions. However, the authors highlight that the association of HIV infection with motor neuron diseases is rare, and ALS retroviral pathogenesis is not well defined. Verma $e t$ al.51 described two cases of ALS in patients with HIV infection. Both diagnosed the infection and further presented weakness in the bulbar musculature and limbs. On the other hand, the authors demonstrated that in this type of association the disease varied in certain aspects in relation to those observed in the classical form: patients are younger, there was no inexorable progression, it decreased after the institution of antiviral therapy and there was evidence of inflammatory response in the central nervous system. Finally, a new 
association was reported by Akhvlediani et $a l ., 55$ who described the case of a patient with a history of 9 years of hepatitis $\mathrm{C}$, with progressive weakness and right arm atrophy. No other case of HCV infection and ALS was reported, but further investigation is necessary, because it can be a new possible association.

\section{Physical activities}

Studies with workers and former athletes who developed ALS associated the history of physical activity to the emergence of the disease, suggesting the hypothesis that vigorous physical activity may be a predisposing factor for developing the disease. 56 This is justified by the fact that physical practice leads to an increase in oxidative stress or by an excitotoxicity by glutamate. Physical activity may alter the balance between the formation and removal of free radicals, leading to oxidative stress, in addition to possibly leading to an overstimulation of motor neurons, with resulting neuronal death. ${ }^{57} \mathrm{~A}$ study performed by Longstreth $\mathrm{Jr}$ et al.58 aimed at assessing the association between the history of physical activity and ALS diagnosis. The subjects were submitted to an interview containing information on physical activity performed before the ALS diagnosis, during work or performed during free time. The authors concluded that physical activity does not seem to be a significant risk factor for ALS, but they highlight the necessity of more studies on the field for a better preventive approach in patients with this disorder. Veldink et al.57 conducted a study with the objective of assessing whether physical activity during work or free time is associated with the increase in the risk of developing ALS and determining the association between physical activity and the duration or age of onset of the disease. Questionnaires were applied in order to identify individual characteristics, including anthropometric features, sex and age, and on the activity performed. In conclusion, no strong association between the practice of physical activity and the risk of developing ALS was identified, since the effects of the activity on the disease were not totally clarified. Soccer is frequently associated with ALS. Wicks et al. ${ }^{59}$ reported 3 cases of soccer players who developed the disease simultaneously. The three patients started to present the symptoms around the 50 years of age, but in different segments (left foot, right leg and higher left limb). All of them performed another kind of occupational activity including chemical exposure, as electrician or engineer. Two athletes had a previous history of smoking, and they were all alcohol drinkers. The three players played soccer for at least 10 years, and only one reported to usually head the ball. The authors highlighted that, although it seems a coincidence, these athletes presented similar clinical histories, being exposed to the same risk factors. However, for this reason, it cannot be concluded that these cases are due only to soccer playing, but that it is strongly associated to the development of this morbidity. Some explanations for this fact include the habit of heading the ball and the use of pesticides in the field.

\section{Conclusion}

From what was exposed, it can be perceived that there is still no consensus on ALS's etiology. Researches show evidence of intoxication by heavy metals, environmental and occupational causes, genetic mutations (superoxide dismutase 1), certain viral infections and the performance of rigorous physical activity for the development of the disease. Due to this great diversity of possible causing agents for ALS, new researches are necessary to elucidate possible etiologies for a better approach to the patients, promoting preventive programs for the disease, optimizing functions and improving the life quality of the patients.

\section{References}

1. Rowland LP, Shneider NA. Amyotrophic Lateral Sclerosis. N Engl J Med 2001;22: 1688-700.

2. Wijesekera LC, Leigh PN. Amyotrophic lateral sclerosis. Orphanet J Rare Dis 2009; $4: 3$.

3. Rothstein JD. Current Hypotheses for the Underlying Biology of Amyotrophic Lateral Sclerosis. Ann Neurol 2009;65:S3-9.

4. Aguilar JLG, Echaniz-Laguna A, Fergani A, et al. Amyotrophic lateral sclerosis: all roads lead to Rome. Journal of Neurochemistry 2007;101:1153-60.

5. Brown-Jr RH. Amyotrophic Lateral Sclerosis and Other Motor Neuron Diseases. In: Fauci AS, Braunwald E, Kasper DL, Hauser SL, Longo DL, Jameson JL, et al. Harrison's Principles of Internal Medicine. New York: McGraw Hill; 2008. p. 2572-576.

6. Bruijn LI, Miller TM, Cleveland DW. Unraveling the mechanisms involved in motor neuron degeneration in ALS. Annu Rev Neurosci 2004;27:723-49.

7. Lacomblez L, Bensimon G, Leigh PN, et al. Dose-ranging study of riluzole in amyotrophic lateral sclerosis. Amyotrophic Lateral Sclerosis/Riluzole Study Group II. Lancet 1996;347:1425-31.

8. Oliveira ASB, Pereira RDB. Amyotrophic Lateral Sclerosis (ALS): Three letters that change the people's life for ever. Arq Neuropsiquiatr 2009;67:750-82.
9. Rosen DR, Siddique T, Patterson D, et al. Mutations in $\mathrm{Cu} / \mathrm{Zn}$ superoxide dismutase gene are associated with familial amyotrophic lateral sclerosis. Nature 1993;362:59-62.

10. Cluskey S, Ramsden DB. Mechanisms of neurodegeneration in amyotrophic lateral sclerosis. Mol Pathol 2001;54:386-92.

11. Wicklund MP. Amyotrophic Lateral Sclerosis: Possible Role of Environmental Influences. Neurol Clin 2005;23:461-84.

12. Orsini M, De Freitas MRG, Oliveira ASB, et al. Esclerosis lateral amiotrófica esporádica de inicio juvenil. Rev Neurol 2010;50: 442-4.

13. Cozzolino M, Ferri A, Carrí MT. Amyotrophic Lateral Sclerosis: From Current Developments in the Laboratory to Clinical Implications. Antioxid Redox Signal 2008;10:405-43.

14. Brooks BR, Sanjak M. Disease-modifying drug therapies. Amyotroph Lateral Scler Other Motor Neuron Disord 2004;5:68-75.

15. Richard OW, Russell JM, Ross M. A systematic review of antioxidant treatment for amyotrophic lateral sclerosis/motor neuron disease. ALS 2008;9:195-211.

16. Mitsumoto H, Chad DA, Pioro EP. Amyotrophic lateral sclerosis. Contemporary neurology series. Oxford University Press. edition 1; vol.49, 1998.

17. Barnham KJ, Masters CL, Bush AI. Neurodegenerative diseases and oxidative stress. Nat Rev Drug Discov 2004;3:205-14.

18. Stanich P, Pereira AML, Chiappetta AL, et al. Suplementação nutricional em pacientes com doença do neurônio motor/ esclerose lateral amiotrófica. Rev Bras Nut Clin 2004;19:70-77.

19. Jackson C, Gronseth G, Rosenfeld J, et al. Randomized double-blind study of botulinum toxin type B for sialorrhea in ALS patients. Muscle Nerve 2009;39:137-43.

20. Shaw CE, Enayat ZE, Chioza BA, et al. Mutations in all five exons of SOD-1 may cause ALS. Ann Neurol 1998;43:390-4.

21. Kwiatkowski TJ, Bosco DA, LeClerc AL, et al. Mutations in the FUS/TLS gene on chromosome 16 cause familial amyotrophic lateral sclerosis. Science 2009;323:12058.

22. Rademakers R, Stewart H, DejesusHernandez M, et al. FUS Gene Mutations in Familial and Sporadic Amyotrophic Lateral Sclerosis. Muscle Nerve 2010;42: 170-6.

23. Harms MM, Miller TM, Baloh RH. TARDBP - Related Amyotrophic Sclerosis. GeneReviews. 2009;Apr 23 [updated 2009 May 28].

24. Kühnlein P, Sperfeld AD, Vanmassenhove B, et al. Two German kindreds with familial amyotrophic lateral sclerosis due to TARDBP mutations. Arch Neurol 2008;65: 
1185-9.

25. Mendonça DM, Martins SC, Higashi R, et al. Neurofilament heavy subunit in cerebrospinal fluid: a biomarker of amyotrophic lateral sclerosis. Amyotroph Lateral Scler 2011;12:144-7.

26. Haramati S, Chapnik E, Sztainberg Y, et al. miRNA malfunction causes spinal motor neuron disease. Proc Natl Acad Sci USA 2010;107:13111-6.

27. Mitchell JD. Amyotrophic lateral sclerosis: toxins and environment. Amyotroph Lateral Scler Other Motor Neuron Disord 2000;1:235-50.

28. Armon C. An evidence-based medicine approach to the evaluation of the role of exogenous risk factors in sporadic amyotrophic lateral sclerosis. Neuroepide miology 2003;22:217-28.

29. Banack SA, Murch SJ. Multiple neurotoxic items in the Chamorro diet link BMAA with ALS/PDC. Amyotroph Lateral Scler 2009;10:34-40.

30. Bradley WG, Mash DC. Beyond Guam: the cyanobacteria/BMAA hypothesis of the cause of ALS and other neurodegenerative diseases. Amyotroph Lateral Scler 2009;10: 7-20.

31. Sieh W, Choi Y, Chapman NH, Craig UK, et al. Identification of novel susceptibility loci for Guam neurodegenerative disease: challenges of genome scans in genetic isolates. Hum Mol Genet 2009;18:3725-38.

32. Weisskopf MG, Morozova N, 0'Reilly EJ, et al. Prospective study of chemical exposures and amyotrophic lateral sclerosis. J Neurol Neurosurg Psychiatry 2009;80:55861.

33. Plato CC, Garruto RM, Galasko D, et al. Amyotrophic lateral sclerosis and parkinsonism-dementia complex of Guam: changing incidence rates during the past 60 years. Am J Epidemiol 2003;157:149-57.

34. Waring SC, Esteban-Santillan C, Reed DM, et al. Incidence of amyotrophic lateral sclerosis and of the parkinsonism-dementia complex of Guam, 1950-1989. Neuroepidemiology 2004;23:192-200.

35. Steele JC, McGeer PL. The ALS/PDC syndrome of Guam and the cycad hypothesis.
Neurology 2008;70:1984-90.

36. Mitchell JD, Borasio GD. Amyotrophic lateral sclerosis. Lancet 2007;369:2031-41.

37. Weisskopf MG, McCullough ML, Morozova N, et al. Prospective Study of Occupation and Amyotrophic Lateral Sclerosis Mortality. Am J Epidemiol 2005;162:114652 .

38. Morahan JM, Pamphlett R. Amyotrophic Lateral Sclerosis and Exposure to Environmental Toxins: An Australian Case-Control Study. Neuroepidemiol 2006;27:130-5.

39. Weisskopf MG, McCullough ML, Calle EE, et al. Prospective Study of Cigarette Smoking and Amyotrophic Lateral Sclerosis. Am J Epidemiol 2004;160:26-33.

40. Fang F, Bellocco R, Hernán MA, Ye Weimin. Smoking, Snuff Dipping and the Risk of Amyotrophic Lateral Sclerosis - A Prospective Cohort Study. Neuroepidemiology 2006;27:217-21.

41. Sutedja NA, Veldink JH, Fischer K, et al. Lifetime occupation, education, smoking, and risk of ALS. Neurology 2007;69:150814.

42. Popat RA, Tanner CM, Van Den Eeden SK, et al. Effect of non-steroidal anti-inflammatory medications on the risk of amyotrophic lateral sclerosis. Amyotroph Lateral Scler 2007;8:157-63.

43. Huynh W, Lam A, Vucic S, et al. Corticospinal tract dysfunction and development of amyotrophic lateral sclerosis following electrical injury. Muscle Nerve 2010;42:288-92.

44. Mitchell JD. Heavy metals and trace elements in amyotrophic lateral sclerosis. Neurol Clin 1987;5:43-60.

45. Kamel F, Umbach DM, Munsat TL, et al. Lead Exposure and Amyotrophic Lateral Sclerosis. Epidemiology 2002;13:311-9.

46. Oh S, Kim EA, Lee S, et al. A case of amyotrophic lateral sclerosis in electronic parts manufacturing worker exposed to lead. NeuroToxicology 2007;28:324-7.

47. Schwarz S, Husstedt IW, Bertram HP, Kuchelmeister K. Amyotrophic lateral sclerosis after accidental injection of mercury. J Neurol Neurosurg Psychiatry 1996;60:
698.

48. Weihl CC, Lopate G. Motor Neuron Disease Associated with Copper Deficiency. Muscle Nerve 2006;34:789-93.

49. Vinceti M, Bonvicini F, Bergomi M, Malagoli C. Possible involvement of overexposure to environmental selenium in the etiology of amyotrophic lateral sclerosis: a short review. Ann Ist Super Sanità 2010;46:279-83.

50. Ravits J. Sporadic amyotrophic lateral sclerosis: A hypothesis of persistent (nonlytic) enteroviral infection. Amyotroph Lateral Scler 2005;6:77-87.

51. Verma A, Berger JR. ALS syndrome in patients with HIV-1 infection. J Neurol Sc 2006;240:59-64.

52. Berger MM, Kopp N, Vital C, et al. Detection and cellular localization of enterovirus RNA sequences in spinal cord of patients with ALS. Neurology 2000;54: 20-5.

53. Jubelt B, Berger JR. Does viral disease underlie ALS?: Lessons from the AIDS pandemic. Neurology 2001;57:945-6.

54. Zoccolella S, Carbonarab S, Minerva D, et al. A case of concomitant amyotrophic lateral sclerosis and HIV infection. European Journal of Neurology 2002;9:177-85.

55. Akhvlediani T, Kvirkvelia N, Shakarishvili $\mathrm{R}$, Tsertsvadze T. ALS-like syndrome in the patient with chronic hepatitis $\mathrm{C}$. Georgian Med News 2009;70-1.

56. Strickland D, Smith SA, Dolliff G, et al. Physical activity, trauma, and ALS: a casecontrol study. Acta Neurol Scand 1996;94: 45-50.

57. Veldink JH, Kalmijn S, Groeneveld GJ, et al. Physical activity and the association with sporadic ALS. Neurology 2005; 64:241-5. 58. Longstreth Jr WT, McGuire V, Kopsell TD, et al. Risk of Amyotrophic Lateral Sclerosis and History of Physical Activity - A Population-Based Case-Control Study. Arch Neurol 1998;55:201-6.

59. Wicks P, Ganesalingham J, Collin C, et al. Three soccer playing friends with simultaneous amyotrophic lateral sclerosis. Amyotroph Lateral Scler 2007;8:177-9. 